\title{
DRAFT
}

\section{Discrete Geometry on Red and Blue Points in the Plane - A Survey -}

Atsushi Kaneko

M. Kano

\begin{abstract}
In this paper, we give a short survey on discrete geometry on red and blue points in the plane, most of whose results were obtained in the past decade. We consider balanced subdivision problems, geometric graph problems, graph embedding problems, Gallai-type problems and others.
\end{abstract}

\section{Notation and Definitions}

In this paper, we give a short survey on discrete geometry on red and blue points in the plane, most of whose results were obtained in the past decade. We consider two disjoint sets $R$ and $B$ of red points and of blue points in the plane, respectively, such that no three points of $R \cup B$ lie on the same line. Throughout this paper, $R$ and $B$ always denote the sets mentioned above unless noted otherwise.

We begin with some notation and definitions, which will be used throughout this paper. A (directed) line $l$ trisects the plane into three pieces: $l$, right $(l)$ and $\operatorname{left}(l)$, where $\operatorname{right}(l)$ and $l$ eft $(l)$ denote the open half-planes which are on the right side and on the left side of $l$, respectively (Figure 1). Let $r_{1}$ and $r_{2}$ be two rays emanating from the same point $p$. Then we denote by $\operatorname{right}\left(r_{1}\right) \cap$ left $\left(r_{2}\right)$ the open region that is swept by the ray being rotated clockwise around $p$ from $r_{1}$ to $r_{2}$ (Figure 1). The open region left $\left(r_{1}\right) \cap \operatorname{right}\left(r_{2}\right)$ is similarly defined. Then $r_{1} \cup r_{2}$ trisects the plane into three pieces: $r_{1} \cup r_{2}$ and two open regions right $\left(r_{1}\right) \cap \operatorname{left}\left(r_{2}\right)$ and left $\left(r_{1}\right) \cap \operatorname{right}\left(r_{2}\right)$. If the internal angle $\angle r_{1} p r_{2}=\angle r_{1} r_{2}$ of $\operatorname{right}\left(r_{1}\right) \cap \operatorname{left}\left(r_{2}\right)$ is less than $\pi$, then we call right $\left(r_{1}\right) \cap \operatorname{left}\left(r_{2}\right)$ the wedge defined by $r_{1}$ and $r_{2}$, and denote it by wedge $\left(r_{1} r_{2}\right)$, wedge $\left(r_{2} r_{1}\right)$, wedge $\left(r_{1} p r_{2}\right)$ or wedge $\left(r_{2} p r_{1}\right)$.
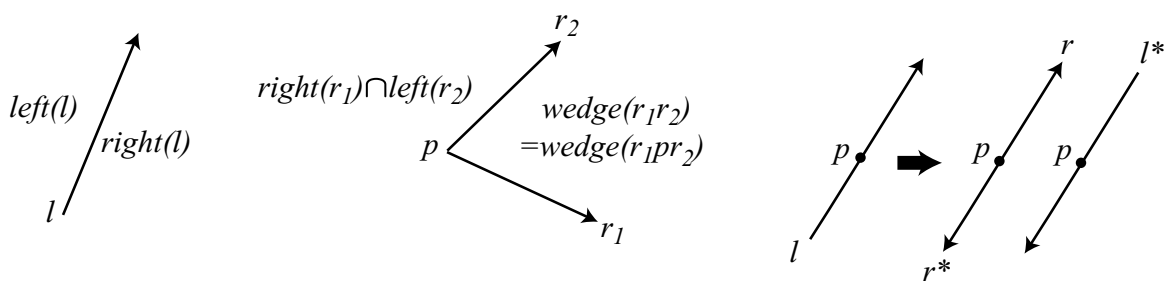

Figure 1: Open regions $\operatorname{right}(l)$, left $(l), \operatorname{left}\left(r_{1}\right) \cap \operatorname{right}\left(r_{2}\right)$ and wedge $\left(r_{1} r_{2}\right)=\operatorname{wedge}\left(r_{1} p r_{2}\right)$, the rays $r$ and $r^{*}$, and the line $l^{*}$.

Given a point $p$ and a line $l$ passing through $p$, let $l^{*}$ denote the line lying on $l$ and having the opposite direction of $l$. We define the rays $r$ and $r^{*}$ to be the two rays emanating from $p$ and lying on the line $l$, while having the direction of $l$ and $l^{*}$ respectively (Figure 1 ). Conversely, given a ray $r$, we can define $r^{*}$, $l$ and $l^{*}$.

For a set $X$ of points in the plane, let $\operatorname{conv}(X)$ denote the convex hull of $X$, which is the smallest convex set containing $X$. For convenience, a region in the plane whose boundary consists of straightline segments is called a polygon even if it is an infinite region. For example, Figure 2 (b) illustrates a subdivision of the plane into seven convex polygons. 


\section{Balanced Subdivisions}

In this section we consider problems of subdivisions of the plane into convex polygons such that (i) each polygon contains exactly $a$ red points and $b$ blue points, (ii) each polygon contains $a n_{i}$ red points and $b n_{i}$ blue points, or (iii) polygons satisfy other prescribed conditions, where $a$ and $b$ are constant positive integers independent of polygons and $n_{i}$ is a positive integer depending on each polygon. A famous classical theorem on this problem is the following Ham-sandwich Theorem.

Theorem 2.1 (Ham-sandwich Theorem [17]). For given $R$ and $B$, there exists a line $l$ such that $|\operatorname{left}(l) \cap R|=|\operatorname{right}(l) \cap R|,|l \cap R| \leq 1,|\operatorname{left}(l) \cap B|=|\operatorname{right}(l) \cap B|$ and $|l \cap B| \leq 1$ (Figure 2 (a)). If both $|R|$ and $|B|$ are even, then $l$ passes through no red points and no blue points, and thus $l$ bisects $R$ and B. Moreover, there exists a linear time $O(|R|+|B|)$ algorithm for finding line l [31].

The line $l$ given in the above Ham-sandwich Theorem is called a bisector line, and the subdivision of the plane by a bisector line is called a Ham-sandwich cutting.

Before considering balanced subdivisions, let us give a result on balanced undirected lines. When $|R|=|B|$, an undirected line $l$ is said to be balanced if each open half-plane bounded by $l$ contains precisely the same number of red points as blue points. Then if $|R|=|B|$, an undirected bisector line is balanced. The following theorem gives an lower bound of the number of balanced undirected lines, and thus settles a conjecture of G. Baloglou.

Theorem 2.2 (Pach and Pinchasi [36]). If $|R|=|B|=n$, then there exist at least $n$ balanced undirected lines each of which passes through one red point and one blue point.

Note that if $|R|=|B|=n$ and $R$ and $B$ are separated by a line, then there exists exactly $n$ balanced undirected lines determined by them, and so the above lower bound $n$ is best possible.

The following theorem, which was conjectured in [21] and proved for $a=1,2$ in [21] and [26], was completely proved by Bespamyatnikh, Kirkpatrick and Snoeyink [7], Sakai [39] and by Ito, Uehara and Yokoyama [19] independently. Note that for $g=2$, it is equivalent to the Ham-Sandwich Theorem above.

Theorem 2.3 (The Equitable Subdivision Theorem [7], [39], [19]). Let $a \geq 1, b \geq 1$ and $g \geq 2$ be integers. If $R$ contains ag red points and $B$ contains bg blue points, then there exists a subdivision $X_{1} \cup X_{2} \cup \cdots \cup X_{g}$ of the plane into $g$ disjoint convex polygons such that every $X_{i}$ contains exactly a red points and $b$ blue points (Figure $2(b)$ ).

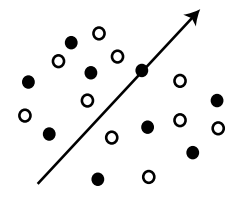

(a)

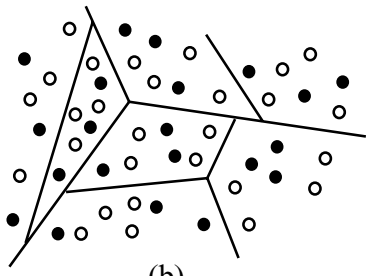

(b)

Figure 2: (a) A Ham-sandwich cutting; (b) An equitable subdivision.

Such a subdivision of the plane is called an equitable subdivision of the plane. An algorithm for finding an equitable subdivision of the plane with time complexity $O\left(n^{4 / 3} \log ^{3} n \log g\right)$, where $n=(a+b) g$, was obtained by Bespamyatnikh, Kirkpatrick and Snoeyink [7].

Theorem 2.4 (Kaneko, Kano and Suzuki [27]). Let $a \geq 1, g \geq 0$ and $h \geq 0$ be integers such that $g+h \geq 1$. If $R$ contains ag $+(a+1) h$ red points and $B$ contains $(a+1) g+$ ah blue points, then there exists a subdivision $X_{1} \cup \cdots \cup X_{g} \cup Y_{1} \cup \cdots \cup Y_{h}$ of the plane into $g+h$ disjoint convex polygons such that every $X_{i}(1 \leq i \leq g)$ contains exactly a red points and $a+1$ blue points and every $Y_{j}(1 \leq j \leq h)$ contains exactly $a+1$ red points and a blue points (Figure $3(b)$ ). 


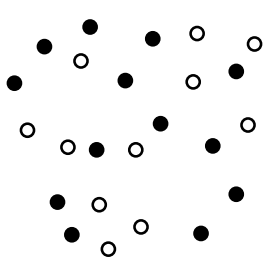

(a)

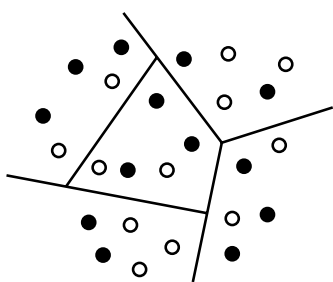

(b)

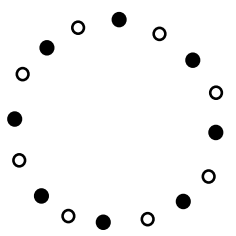

(c)

Figure 3: (a), (b) $R \cup B$ and a subdivision from Theorem 2.4; (c) A configuration having no subdivision with $a=1, b=3$ and $g=h=2$..

The above theorem naturally suggests the following problem. Let $a \geq 1$ and $b \geq a+2$ be integers. If $R$ contains $a g+b h$ red points and $B$ contains $b g+a h$ blue points, then does there exist a subdivision $X_{1} \cup \cdots \cup X_{g} \cup Y_{1} \cup \cdots \cup Y_{h}$ of the plane into $g+h$ disjoint convex polygons such that every $X_{i}$ contains exactly $a$ red points and $b$ blue points and every $Y_{j}$ contains exactly $b$ red points and $a$ blue points? However this statement does not hold since for a configuration in which $a g+b g$ red points and $b g+a g$ blue points alternately lie on a circle, there exists no subdivision mentioned above (Figure 3 (c)).

The Ham-sandwich Theorem, the Equitable Subdivision Theorem and Theorem 2.4 may be applied to some problems on geometry on red and blue points in the plane. We give such an example.

It is well-known ([30]) that given $R \cup B$ with $|R|=|B|$, there exists a non-crossing geometric perfect matching on $R \cup B$, that is, there exist $|R|$ disjoint straight-line segments which connect red points and blue points and have no crossings. This result can be easily proved by using the Ham-sandwich Theorem and induction on $|R|$. That is, if $|R|$ is odd, then a straight-line segment lying on the bisector line $l$ whose endpoints are the red point and the blue point on $l$ is chosen to be an element of a perfect matching, and it is obvious how to apply the induction hypotheses in the case where $|R|$ is even.

We can generalize this problem to the following problem, which is called a non-crossing geometric alternating path-covering problem (Figure 4 (a)). We denote by $P_{k}$ the path with $k$ vertices and length $k-1$ that passes through red points and blue points alternately. For a constant integer $n$ and for given $R$ and $B$, do there exist disjoint non-crossing geometric alternating paths $P_{n}$ which cover $R \cup B$ ? If $P_{n}=P_{2 m}$ and $|R|=|B|=m g$, then we first apply the Equitable Subdivision Theorem to $R \cup B$, and obtain the equitable subdivision of the plane in which each convex polygon contains $m$ red points and $m$ blue points. Thus if we can show that for every arrangement of $m$ red points and $m$ blue points in the plane in general position, there exists a non-crossing geometric alternating path $P_{2 m}$ that passes through all these red and blue points, the above problem is affirmatively solved.

When we consider the above problem with path $P_{2 m+1}, R$ must contain $m g+(m+1) h$ red points and $B$ must contain $(m+1) g+m h$ blue points, where integers $g$ and $h$ correspond to the numbers of paths $P_{2 m+1}$ whose both endpoints are blue points or red points, respectively. In this case, we first obtain a subdivision of the plane by Theorem 2.4, and if we can show that for every arrangement of $m$ red points and $m+1$ blue points in the plane in general position, there exists a non-crossing geometric alternating path $P_{2 m+1}$ that passes through all these red and blue points, then the above problem is affirmatively solved.

It is shown that if $R$ and $B$ are separated by a line and $|R|=|B|$, then there exists a non-crossing geometric alternating path that passes through all the points in $R \cup B$ [1]. On the other hand, as we show in Figure 4 (c), there exists a configuration consisting of six red points and seven blue points in the plane for which there exists no non-crossing geometric alternating path $P_{13}$ that passes through all these 13 points. Considering this observation, we obtain the following theorem, which is easily proved for $2 \leq n \leq 7$ by the Ham-sandwich Theorem (Figure 5 (b)).

Theorem 2.5 ([27]). Suppose that $2 \leq n \leq 12$. Then for any given $\lfloor n / 2\rfloor$ red points and $\lceil n / 2\rceil$ blue points in the plane in general position, there exists a non-crossing geometric alternating path $P_{n}$ that passes through all these red and blue points. 


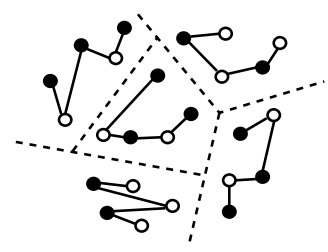

(a)

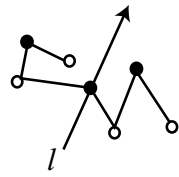

(b)

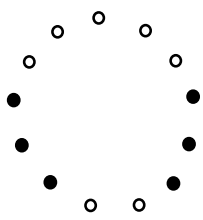

(c)

Figure 4: (a) A non-crossing geometric alternating $P_{5}$-covering (b) A non-crossing geometric alternating $P_{7}$ and a bisector line $l$; (c) A configuration having no non-crossing geometric alternating path.

We now consider balanced subdivisions of the plane, in which the numbers of red points and blue points are different depending on convex polygons.

Theorem 2.6 ([28]). Let $m \geq 1, n \geq 1$ and $g \geq 2$ be integers such that $m+2 n=2 g+1$. If $R$ contains $2 g+1$ red points and $B$ contains $(2 g+1) b$ blue points, then there exists a subdivision $X_{1} \cup X_{2} \cup \cdots \cup X_{m+n}$ of the plane into $m+n$ disjoint convex polygons such that every $X_{i}(1 \leq i \leq m)$ contains one red point and $b$ blue points and every $X_{j}(m<j \leq m+n)$ contains two red points and $2 b$ blue points (Figure $\left.5(a)\right)$.

It is easy to see that if the above theorem holds for $m=1$, then the theorem holds for every $m$, since each convex polygon containing two red points and $2 b$ blue points can be bisected by the Ham-sandwich Theorem.

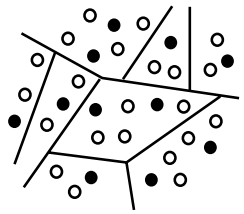

(a)

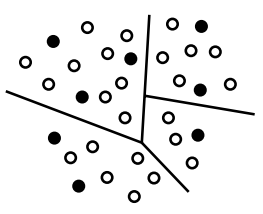

(b)

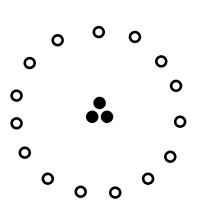

(c)

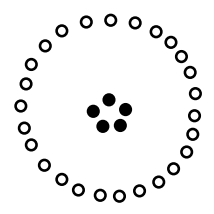

Figure 5: (a) A balanced subdivision from Theorem 2.6; (b) A balanced subdivision from Theorem 2.7; (c) Configurations having no balanced subdivisions with $\left(n_{1}, n_{2}\right)=(2,1)$ and $\left(n_{1}, n_{2}\right)=(3,2)$, respectively.

Theorem 2.7 ([24]). Let $2 \leq n \leq 8,1 \leq b$ and $2 \leq g$ be integers. If $R$ contains $n$ red points and $B$ contains bn blue points, then for every integer-partition $n=n_{1}+n_{2}+\ldots+n_{g}$ such that $1 \leq n_{i} \leq n / 2$ for every $1 \leq i \leq g$, there exists a subdivision $X_{1} \cup X_{2} \cup \cdots \cup X_{g}$ of the plane into $g$ convex polygons such that each $X_{i}(1 \leq i \leq g)$ contains exactly $n_{i}$ red points and bn $n_{i}$ blue points.

Figure 5 (b) shows a balanced subdivision with $\left(n_{1}, n_{2}, n_{3}, n_{4}\right)=(3,2,2,1)$, Figure 5 (c) gives two configurations $R \cup B$ having no balanced subdivision with $\left(n_{1}, n_{2}\right)=(2,1)$ and $\left(n_{1}, n_{2}\right)=(3,2)$, respectively. Similarly, if $n / 2<n_{1}<n$, then there exist configurations $R \cup B$ having no balanced subdivision with $n=n_{1}+n_{2}$. Based on Theorems 2.6 and 2.7 and by some other configurations given in [24], we propose the following conjecture.

Conjecture $2.8([\mathbf{2 4}])$. Let $n=n_{1}+n_{2}+\cdots+n_{g}$ be an integer partition such that $1 \leq n_{i} \leq n / 3$ for every $1 \leq i \leq g$. If $|R|=$ an and $|B|=b n$, then there exists a subdivision $X_{1} \cup X_{2} \cup \cdots \cup X_{g}$ of the plane into $g$ disjoint convex polygons such that each $X_{i}(1 \leq i \leq g)$ contains exactly an $n_{i}$ red points and bn $n_{i}$ blue points.

The following theorem gives a subdivision, which might be called a semi-balanced subdivision.

Theorem 2.9 ([26]). Let $m \geq 0$ be an integer, and let $R=R_{1} \cup R_{2}$ be a partition of $R$ with $\left|R_{1}\right|=s \geq 0$ and $\left|R_{2}\right|=t \geq 0$. If $|B|=m s+(m+1) t$, then there exists a subdivision $X_{1} \cup X_{2} \cup \cdots \cup X_{s+t}$ of the 
plane into $s+t$ convex polygons such that each $X_{i}(1 \leq i \leq s)$ contains one red point of $R_{1}$ and $m$ blue points and each $X_{j}(s<j \leq s+t)$ contains one red point of $R_{2}$ and $m+1$ blue points.

We can consider a generalization of the above theorem by taking a partition $R=R_{1} \cup R_{2} \cup R_{3}$ and three integers $m, m+1, m+2$. But this generalization does not hold as shown in [26].

Bárány and Matoušek [5] consider balanced subdivisions of the plane by $k$-fan, and obtain similar results. Though they consider $t$ Borel probability measure in the plane, here we consider $t$ sets $X_{1}, X_{2}, \ldots, X_{t}$ of points in the plane in general position. A $k$-fan is defined to be a point $x$ in the plane and $k$ rays emanating from $x$, and by this $k$-fan, the plane is subdivided into $k$ open regions $W_{1} \cup W_{2} \cup \cdots \cup W_{k}$, one of which may not be convex (Figure 6 (a)). A $k$-tuple of parallel lines is also considered to be a $k$-fan (this is a limit case for $x$ receding to infinity). For a vector $\alpha=\left(\alpha_{1}, \alpha_{2}, \ldots, \alpha_{k}\right)$ of non-negative real numbers whose components sum up to 1 , we say that a $k$-fan simultaneously $\alpha$-partitions $X_{1}, X_{2}, \ldots, X_{t}$ if

$$
\left|X_{i} \cap W_{j}\right|=\alpha_{j}\left|X_{i}\right| \quad \text { for all } 1 \leq i \leq t \text { and } 1 \leq j \leq k .
$$

Note that when we consider a simultaneous $\alpha$-partition, we always assume that $\alpha_{j}\left|X_{i}\right|$ is an integer for all $i$ and $j$. In particular, a $k$-fan simultaneously $\left(\frac{1}{k}, \frac{1}{k}, \ldots, \frac{1}{k}\right)$-partitions $X_{1}, X_{2}, \ldots, X_{t}$ if

$$
\left|X_{i} \cap W_{j}\right|=\frac{1}{k}\left|X_{i}\right| \quad \text { for all } 1 \leq i \leq t \text { and } 1 \leq j \leq k .
$$

The following theorem was proved by using topological methods, and so it would be interesting to have an elementary proof for it.

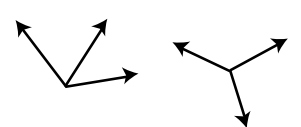

(a)

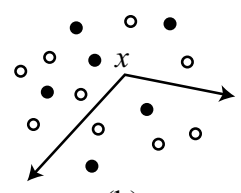

(b)

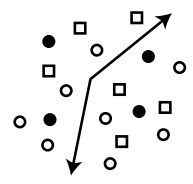

(c)

Figure 6: (a) Two 3-fans; (b) $R$ and $B$ is simultaneously $\left(\frac{1}{3}, \frac{2}{3}\right)$-partitioned by a 2 -fan with center $x$. (c) $R, B$ and $Y$ is simultaneously $\left(\frac{1}{2}, \frac{1}{2}\right)$-partitioned by a 2 -fan.

Theorem 2.10 (Bárány and Matoušek [5]). Let $R, B, Y$ be three disjoint sets of red points, blue points and yellow points in the plane, respectively, such that no three points of $R \cup B \cup Y$ lie on the same line. Then the following statements hold.

(i) For any $\alpha=\left(\alpha_{1}, \alpha_{2}\right)$ and any point $x$ in the plane, $R$ and $B$ can be simultaneously $\alpha$-partitioned by a 2-fan with center $x$. 2-fan.

(ii) For $\alpha=\left(\frac{1}{2}, \frac{1}{2}\right)$ and for $\alpha=\left(\frac{2}{3}, \frac{1}{3}\right)$, any $R, B$ and $Y$ can be simultaneously $\alpha$-partitioned by a

(iii) Every $R$ and $B$ can be simultaneously $\left(\frac{1}{3}, \frac{1}{3}, \frac{1}{3}\right)$-partitioned by a 3-fan, or $\left(\frac{1}{2}, \frac{1}{4}, \frac{1}{4}\right)$-partitioned by a 3-fan. They can also be $\left(\frac{2}{5}, \frac{1}{5}, \frac{1}{5}, \frac{1}{5}\right)$-partitioned by a 4-fan.

(iv) For any $k \geq 3$ and any $\alpha$, there exist $R, B$ and $Y$ that can not be simultaneously $\alpha$-partitioned by a $k$-fan.

Theorem 2.11 (Bárány and Matoušek [6]). Every $R$ and $B$ can be simultaneously $\left(\frac{1}{4}, \frac{1}{4}, \frac{1}{4}, \frac{1}{4}\right)$ - partitioned by a \&-fan.

\section{Proof Techniques}

In this section we give some theorems and lemmas which are used in the proofs of some theorems on balanced subdivisions from Section 2 and other geometric problems. We begin with the following lemma, whose distinct proofs can be found in [7] and [24]. 
Lemma 3.1 (The Intermediate Line Lemma). Let $k \geq 0$ be an integer. If there exist two lines $l_{1}$ and $l_{2}$ such that $\mid$ left $\left(l_{1}\right) \cap R|=|$ left $\left(l_{2}\right) \cap R \mid$ and $\mid$ left $\left(l_{1}\right) \cap B|\leq k \leq| \operatorname{left}\left(l_{2}\right) \cap B \mid$, then there exists a line $l_{3}$ such that $\mid$ left $\left(l_{3}\right) \cap R|=|$ left $\left(l_{1}\right) \cap R \mid$, $\mid$ left $\left(l_{3}\right) \cap B \mid=k$ and $l_{3}$ passes through no point in $R \cup B$.

We now prove the Ham-sandwich Theorem by using the above Intermediate Line Lemma. For simplicity, we assume that $|R|$ and $|B|$ are even. It is easy to find a line $l$ with $|\operatorname{left}(l) \cap R|=|\operatorname{right}(l) \cap R|$, which implies $|\operatorname{left}(l) \cap R|=\left|\operatorname{left}\left(l^{*}\right) \cap R\right|=|R| / 2$. Without of loss generality, we may assume that $|\operatorname{left}(l) \cap B|<|B| / 2$. Then

$$
|\operatorname{left}(l) \cap B|<|B| / 2<\left|\operatorname{left}\left(l^{*}\right) \cap B\right| .
$$

Hence by the Intermediate Line Lemma, there exists a line $l_{3}$ satisfying $\left|\operatorname{left}\left(l_{3}\right) \cap R\right|=|R| / 2$ and $\left|\operatorname{left}\left(l_{3}\right) \cap B\right|=|B| / 2$, and thus $l_{3}$ is the desired bisector line.

The following theorem, called the 3-cutting Theorem, is very powerful when we consider balanced subdivision problems and other related problems. This theorem was originally proved by Bespamyatnikh, Kirkpatrick and Snoeyink [7] under the assumption that

$$
\frac{g_{1}}{h_{1}}=\frac{g_{2}}{h_{2}}=\frac{g_{3}}{h_{3}} .
$$

But as mentioned in [24], this condition can be removed without changing the arguments in the proof given in [7]. Similar results, which seems to be essentially equivalent to the following 3-cutting Theorem, were obtained in [39] and [19], respectively.

Theorem 3.2 (The 3-cutting Theorem). Let $g_{1}, g_{2}, g_{3}, h_{1}, h_{2}, h_{3}$ be positive integers such that $|R|=$ $g_{1}+g_{2}+g_{3}$ and $|B|=h_{1}+h_{2}+h_{3}$. Suppose that one of the following statements (i) and (ii) holds:

(i) For every integer $i \in\{1,2,3\}$ and for every line l with $\mid$ left $(l) \cap R\left|=g_{i},\right|$ left $(l) \cap B \mid<h_{i}$.

(ii) For every integer $i \in\{1,2,3\}$ and for every line $l$ with $\mid$ left $(l) \cap R \mid=g_{i}$, $\mid$ left $(l) \cap B \mid>h_{i}$.

Then there exists three rays emanating from a common point such that the three open regions $W_{i}(1 \leq$ $i \leq 3)$ defined by these three rays are wedges, and each wedge $W_{i}(1 \leq i \leq 3)$ contains exactly $g_{i}$ red points and $h_{i}$ blue points. Moreover, one of the three rays can be chosen to be a vertically downward ray (Figure 7).

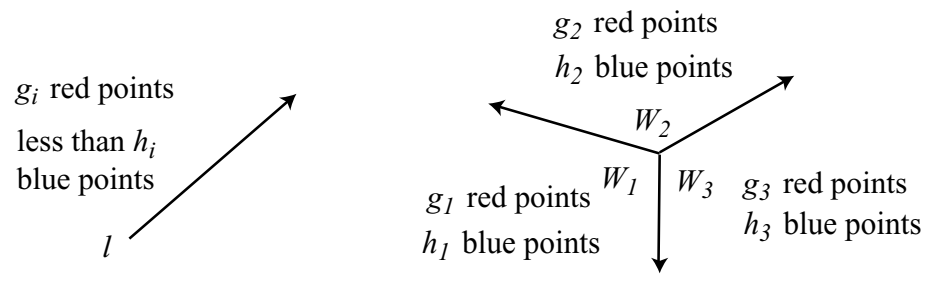

Figure 7: A subdivision given in the 3-cutting theorem.

Now we prove the Equitable Subdivision Theorem using Lemma 3.1 and the 3-cutting Theorem.

Proof of the Equitable Subdivision Theorem [7]. We prove the theorem by induction on $g$. Let $m$ be an integer such that $1 \leq m \leq g-1$. If there exists a line $l$ such that left $(l)$ contains exactly am red points and $b m$ blue points, then by applying the inductive hypotheses to left $(l)$ and $\operatorname{right}(l)$ respectively, we can obtain the desired subdivision of the plane. Hence we may assume that for every line $l$ with $|\operatorname{left}(l) \cap R|=a m$, it follows that $|\operatorname{left}(l) \cap B| \neq b m$. By Lemma 3.1, this fact implies that for every line $l$ with $\mid$ left $(l) \cap R \mid=a m$, either always $\mid$ left $(l) \cap B \mid<b m$ holds or always $|\operatorname{left}(l) \cap B|>b m$ holds. Hence we can define $\operatorname{sign}(m)$ as

$$
\operatorname{sign}(m)=+ \text { if }|\operatorname{left}(l) \cap B|>b m \text { for every line } l \text { with }|\operatorname{left}(l) \cap R|=a m \text {; }
$$


$\operatorname{sign}(m)=-$ if $\mid$ left $(l) \cap B \mid<b m$ for every line $l$ with $\mid$ left $(l) \cap R \mid=a m$.

Without loss of generality, we may assume $\operatorname{sign}(1)=-$ since otherwise by exchanging the colors red and blue, we have $\operatorname{sign}(1)=-$. It is clear that $\operatorname{sign}(1)=-$ implies $\operatorname{sign}(g-1)=+\operatorname{since}|\operatorname{left}(l) \cap R|=a$ and $|\operatorname{left}(l) \cap B|<b$ implies that $\left|\operatorname{left}\left(l^{*}\right) \cap R\right|=(g-1) a$ and $\left|\operatorname{left}\left(l^{*}\right) \cap B\right|>(g-1) b$. Hence there exists an integer $2 \leq k \leq g-1$ such that

$$
\operatorname{sign}(1)=\cdots=\operatorname{sign}(k-1)=-\quad \text { and } \quad \operatorname{sign}(k)=+.
$$

Since $\operatorname{sign}(k)=+$, we have $\operatorname{sign}(g-k)=-$, and hence

$$
\operatorname{sign}(1)=\operatorname{sign}(k-1)=\operatorname{sign}(g-k)=-\quad \text { and } \quad 1+(k-1)+(g-k)=g .
$$

Therefore by the 3-cutting Theorem, the plane can be divided into three wedges $W_{1} \cup W_{2} \cup W_{3}$ such that $W_{1}, W_{2}, W_{3}$ contain $a$ red points and $b$ blue points, $a(k-1)$ red points and $b(k-1)$ blue points, and $a(g-k)$ red points and $b(g-k)$ blue points, respectively. By applying the inductive hypothesis to each $W_{i}$, we can obtain the desired equitable subdivision of the plane. $|R|$.

The next lemma is used in the proofs of Theorems 4.4 and 4.5 , and can be proved by induction on

Lemma 3.3. Let $|R| \geq|B| \geq 2$ and let $p$ be a point in the plane not contained in $\operatorname{conv}(R \cup B)$ such that no three points of $R \cup B \cup\{p\}$ lie on the same line. Suppose that two vertices of $\operatorname{conv}(R \cup B \cup\{p\})$ adjacent to $p$ are blue points. Then there exist two rays $r_{1}$ and $r_{2}$ emanating from $p$ such that (i) each $r_{i}$ passes through exactly one red point, (ii) right $\left(r_{1}\right) \cap$ left $\left(r_{2}\right)$ contains no points in $R \cup B$, and (iii) $\mid\left(\right.$ left $\left.\left(l_{1}\right) \cup l_{1}\right) \cap R|=|$ left $\left(l_{1}\right) \cap B \mid$. (Figure 8).

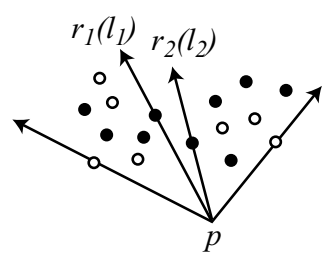

Figure 8: Two rays $r_{1}$ and $r_{2}$ from Lemma 3.3.

\section{Geometric Graphs}

In this section we consider some problems on geometric graphs on red and blue points in the plane. Readers who are interested in geometric graphs in the plane should refer to the book [34] by Pach and Agarwal and the survey [33] by Pach.

A geometric graph is a graph drawn in the plane whose edges are straight-line segments. Let $X$ be a set of points in the plane in general position. Then a geometric spanning tree on $X$, denoted by tree $(X)$, is defined to be a spanning tree on $X$ whose edges are straight-line segments connecting two points in $X$. A geometric Hamiltonian path, path $(X)$, on $X$ and a geometric Hamiltonian cycle, cycle $(X)$, on $X$ are defined analogously (Figure 9 (a),(b)). The geometric complete graph $K(X)$ on $X$ is defined to be a graph whose vertex set is $X$ and whose edge set consists of all the straight-line segments connecting any two points of $X$. Then $\operatorname{tree}(X), \operatorname{path}(X)$ and $\operatorname{cycle}(X)$ are a spanning tree, a Hamiltonian path and a Hamiltonian cycle of $K(X)$, respectively. For two disjoint sets $X$ and $Y$ of points in the plane, we define the geometric complete bipartite graph $K(X, Y)$ as a graph whose vertex set is $X \cup Y$ and whose edge set consists of all the straight-line segments connecting any point in $X$ and any point in $Y$. Thus it follows that the disjoint union $K(X) \cup K(Y) \cup K(X, Y)$ is the geometric complete graph $K(X \cup Y)$.

We first consider the following problem. When we wish to draw red and blue geometric spanning trees $\operatorname{tree}(R)$ and $\operatorname{tree}(B)$ on given $R \cup B$ so that the number of crossings in $\operatorname{tree}(R) \cup \operatorname{tree}(B)$ is as small 
as possible, how do we draw such spanning trees and what is the minimum number of crossings? This problem is solved in the following theorem.

Theorem 4.1 (Tokunaga [42]). Let $\tau(R, B)$ denote the number of unordered pairs $\{x, y\}$ of vertices of $\operatorname{conv}(R \cup B)$ such that one of $\{x, y\}$ is red and the other is blue, and $x y$ is an edge of $\operatorname{conv}(R \cup B)$. Then $\tau(B, R)$ is an even number, and the minimum number of crossings in tree $(R) \cup$ tree $(B)$ among all pairs $(\operatorname{tree}(R)$, tree $(B))$ is equal to

$$
\max \left\{\frac{\tau(R, B)-2}{2}, 0\right\} .
$$

In particular, we can draw red and blue geometric spanning trees without crossings if and only if $\tau(B, R) \leq$ 2 (Figure $9($ a)). Furthermore, the proof gives a polynomial time algorithm for drawing tree $(R) \cup$ tree $(B)$ with minimum number of crossings.

Theorem 4.2 (Tokunaga [42]). For given $R$ and $B$, there exists a pair $($ path $(R)$, path $(B))$ of red and blue geometric Hamiltonian paths such that each edge of path $(R)$ intersects at most one edge of path $(B)$ and vice versa. In particular, the number of crossings in path $(R) \cup$ path $(B)$ is less than or equal to $\min \{|B|,|R|\}-1$ (Figure 9 (b)).

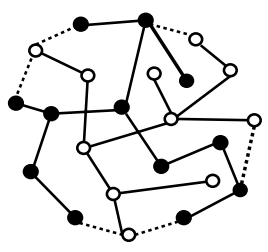

(a)

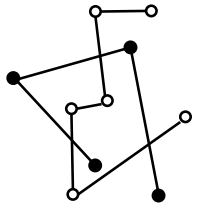

(b)

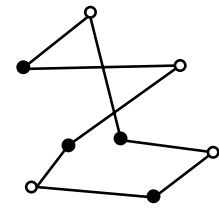

(c)

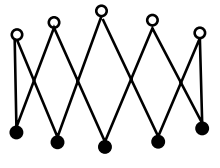

(d)

Figure 9: (a) $\operatorname{tree}(R)$, $\operatorname{tree}(B)$ and $\tau(R, B)=6$; (b) path $(R)$ and path $(B)$; (c) A geometric alternating Hamiltonian cycle with two crossings; (d) A geometric alternating Hamiltonian cycle with $|R|-1$ crossings.

Theorem 4.3 (Dumitrescu and Kaye [11]). Let $n=|R|+|B|$. Then the following two statements hold.

(i) For given $R$ and $B$, there exists a non-crossing matching in $K(R) \cup K(B)$ which covers at least $0.8571 n$ points of $R \cup B$. There exists an algorithm for finding such a matching in $O\left(n^{2}\right)$ time.

(ii) There exists a configuration $R \cup B$ for which every non-crossing matching in $K(R) \cup K(B)$ covers at most $0.9871 n$ points of $R \cup B$.

We next consider geometric alternating spanning graphs on $R \cup B$, whose edges connect red points and blue points, that is, hereafter we deal with spanning subgraphs of $K(R, B)$. For example, a geometric alternating Hamiltonian cycle passes through all the points of $R \cup B$ and through red points and blue points alternately.

Akiyama and Urrutia [4] considered a geometric alternating Hamiltonian cycle on a set of $n$ red points and $n$ blue points lying on the same circle, and gave a $O\left(n^{2}\right)$ time algorithm for finding such a geometric alternating Hamiltonian cycle (if it exists). An upper bound of the number of crossings of geometric alternating Hamiltonian cycle is given in the following theorem.

Theorem 4.4 (Kaneko, Kano, Yoshimoto [25]). For $R$ and $B$ with $|R|=|B|$, there exists a geometric alternating Hamiltonian cycle on $R \cup B$ that has at most $|R|-1$ crossings (Figure 9 (c)). Moreover there exist configurations $R \cup B$ for which this upper bound $|R|-1$ is best possible (Figure $9(d)$ ).

As shown in Theorem 4.1 , for sets $R$ and $B$ with $\tau(R, B) \geq 4$, every $\operatorname{tree}(R) \cup \operatorname{tree}(B)$ contains at least one crossing. On the other hand, we can easily show by induction that for every sets $R$ and $B$, there exists a geometric alternating spanning tree on $R \cup B$ that has no crossings. Namely, we can show 
that $K(R, B)$ contains a spanning tree without crossings. In 1996, Albellanas et al. [1] proved that if $|R|=|B|$, then there exists a non-crossing geometric alternating spanning tree on $R \cup B$ whose maximum degree is at most $O(\log |R|)$. Recently, Kaneko obtained the best upper bound for the maximum degree of the tree mentioned above. In his proof, Lemma 3.3 plays an important role.

Theorem 4.5 (Kaneko [22]). For $R$ and $B$ with $|R|=|B|$, there exists a non-crossing geometric alternating spanning tree on $R \cup B$ whose maximum degree is at most three (Figure $10(a)$ ). This upper bound is sharp.

The following theorem, which was conjectured in [22], has been recently proved by Kaneko.

Theorem 4.6. Let $|R| \leq|B|$ and $k=\lceil|B| /|R|\rceil$. Then there exists a non-crossing geometric alternating spanning tree on $R \cup B$ whose maximum degree is at most $k+2$.

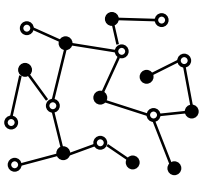

(a)

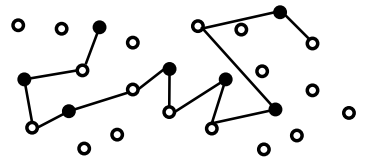

(b)

Figure 10: (a) A non-crossing spanning tree of $K(R, B)$ with maximum degree at most three; (b) A non-crossing Hamiltonian path of $K\left(R, B^{\prime}\right)$ for some $B^{\prime} \subset B$.

As stated in Theorem 4.4, geometric alternating Hamiltonian cycle and path may have many crossings for some $R \cup B$. However, if the number of blue points is much bigger than that of red points, can we then choose a subset $B^{\prime}$ of $B$ such that there exists a non-crossing geometric alternating Hamiltonian path (or cycle) on $R \cup B^{\prime}$ ? The following theorem gives a partial answer to this question on a Hamiltonian path.

Theorem 4.7 (Kaneko and Kano [22]). Let $|R|=n \geq 3$. If $|B| \geq(n+1)(2 n-4)+1$, then we can find a subset $B^{\prime}$ of $B$ with $n$ points such that there exists a non-crossing geometric alternating Hamiltonian path on $R \cup B^{\prime}$ (Figure 10 (b)). Moreover, there exists a configuration of $R \cup B$ with $|B|=\frac{n^{2}}{16}+\frac{n}{2}-1$ for which no such subset $B^{\prime}$ of $B$ exists.

\section{Graph embeddings}

For a graph $G$, we denote by $|G|$ the number of vertices of $G$. Given a planar graph $G$, let $X$ be a set of $|G|$ points in the plane in general position. Then $G$ is said to be line embeddable onto $X$ if $G$ can be embedded in the plane so that every vertex of $G$ corresponds to a point of $X$, every edge corresponds to a straight-line segment, and no two straight-line segments intersect except possibly having a common endpoint. A graph is called an outerplanar graph if it can be embedded in the plane so that every vertex of $G$ lies on the exterior region.

The following theorem is a basic result on line embedding problems. Note that its proof is not found in [14], but Lemma 1 in Section 3 implies this theorem ([42]).

Theorem 5.1 (de Fraysseix, Pach and Pollack [14]). A planar graph $G$ can be line embedded onto an arbitrarily given set $X$ of $|G|$ points in the plane in general position if and only if $G$ is an outerplanar graph.

We now consider a line embedding on red and blue points. Let $G$ be a planar graph with $n$ specified vertices $v_{1}, v_{2}, \ldots, v_{n}$, and $X$ a set of $|G|$ points in the plane in general position which contains $n$ red points $p_{1}, p_{2}, \ldots, p_{n}$ and $|G|-n$ blue points. Then we say that $G$ is strongly line embeddable onto $X$ or $G$ has a strong line embedding onto $X$ if $G$ can be line embedded onto $X$ so that for every $1 \leq i \leq n$, $v_{i}$ corresponds to the red point $p_{i}$ (Figure 11). A tree with one specified vertex $v$ is called a rooted tree 


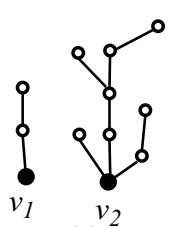

(a)

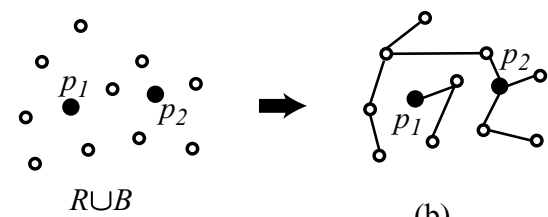

(b)

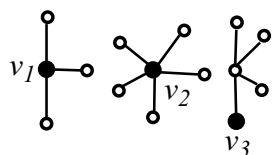

(c)

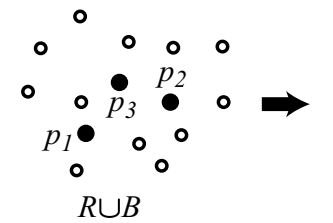

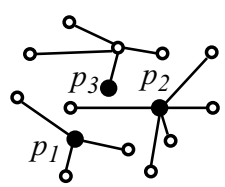

(d)

Figure 11: (a) A rooted forest $F$ with two components; (b) A strong line embedding of $F$; (c) A rooted star forest $G$; (d) A strong line embedding of $G$.

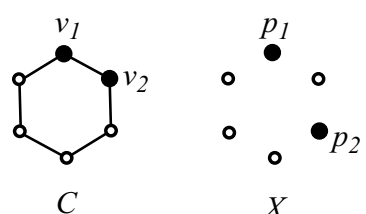

(a)

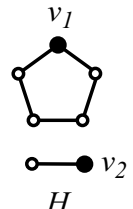

$H$

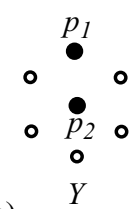

(b)

Figure 12: (a) A cycle $C$ cannot be strongly line embedded onto $X$; (b) A graph $H$ cannot be strongly line embedded onto $Y$.

with root $v$. Given $n$ disjoint rooted trees $T_{i}$ with root $v_{i}, 1 \leq i \leq n$, the union $T_{1} \cup T_{2} \cup \cdots \cup T_{n}$, whose vertex set is $V\left(T_{1}\right) \cup V\left(T_{2}\right) \cup \cdots \cup V\left(T_{n}\right)$ and whose edge set is $E\left(T_{1}\right) \cup E\left(T_{2}\right) \cup \cdots \cup E\left(T_{n}\right)$, is called a rooted forest with roots $v_{1}, v_{2}, \ldots, v_{n}$, which are specified vertices of it.

Before giving our results, we remark that for a cycle $C$ having two specified adjacent vertices and for a set $X$ of $|C|-2$ blue points and two red points in the plane such that all the points of $X$ lie on a circle, and the two red points are not adjacent in $\operatorname{conv}(X), C$ cannot be strongly line embedded onto $X$ (Figure $12(\mathrm{a})$ ). Another such example is given in Figure $12(\mathrm{~b})$, where a graph $H$ consisting of a cycle $C_{5}$ and a path $P_{2}$ cannot be strongly line embedded onto $Y$. Hence, when we consider a strong line embedding problem, we may restrict ourselves to rooted forests.

The following theorem, conjectured by Perles [38], was partially solved by Pach and Töröcsik [37], and completely proved by Ikebe, Perles, Tamura and Tokunaga [20]. A simpler proof of it can be found in Tokunaga [41]. Another related result can be found in [14].

Theorem 5.2. A rooted tree $T$ can be strongly line embedded onto every set of $|T|$ points in the plane in general position containing one red point.

Theorem $5.3([\mathbf{2 3}])$. A rooted forest $F$ consisting of two rooted trees can be strongly line embedded onto every set of $|F|$ points in the plane in general position containing two red points (see Figure $11(a)$, (b)). There exists an $O\left(|F|^{2} \log |F|\right)$ time algorithm for finding a strong line embedding.

It was conjectured that a rooted forest $F$ consisting of three rooted trees can be strongly line embedded onto every set of $|F|$ points in the plane in general position containing three red points ([22]). But a counter-example to this conjecture was found in [29].

A star $K(1, k)(k \geq 1)$ consists of the vertex set $\left\{x, y_{1}, y_{2}, \ldots, y_{k}\right\}$ and the edge set $\left\{x y_{1}, x y_{2}, \ldots, x y_{k}\right\}$, where $x$ is called its center and $y_{i}$ is called its end-vertex. The union of stars is called a star forest, and the union of rooted stars, some of whose roots may be end-vertices, is called a rooted star forest (Figure 11 (c)). 

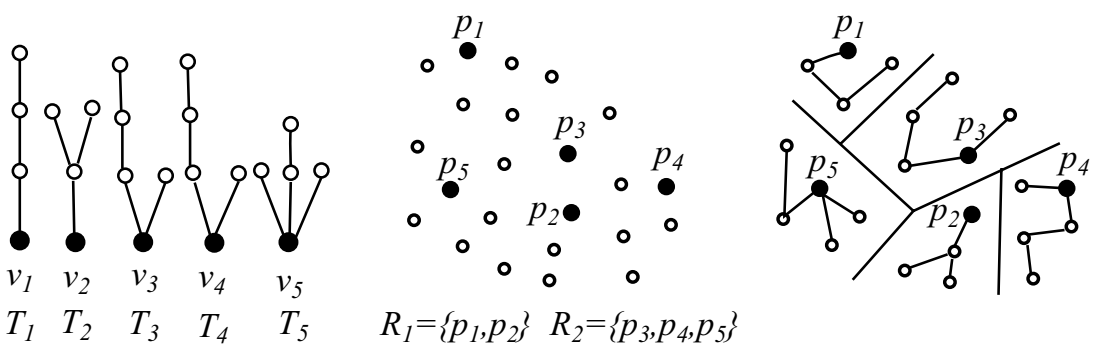

Figure 13: A rooted forest $F$, and its strong line embedding onto $R \cup B$.

Theorem 5.4. A rooted star forest $F$ consisting of $n$ rooted stars can be strongly line embedded onto every set of $|F|$ points in the plane in general position containing $n$ red points (see Figure $11(c),(d)$ ).

By combining Theorem 2.9 and Theorem 5.2, we can obtain the next theorem.

Theorem 5.5. Let $m$ be a positive integer, and let $T_{1}, T_{2}, \cdots, T_{n}$ be $n$ disjoint rooted trees such that $\left|T_{i}\right| \in\{m, m+1\}$ for all $1 \leq i \leq n$. Then the rooted forest $F=T_{1} \cup T_{2} \cup \cdots \cup T_{n}$ can be strongly line embedded onto every set of $|F|$ points in the plane in general position containing $n$ red points (Figure 13).

\section{Gallai-type and Other Problems}

In this section we consider $R \cup B$ not necessarily in general position.

A well-known theorem of Gallai ([16], [40]) states that for any set $S$ of points in the plane all of which do not lie on the same line, there exists a line that passes through exactly two points of $S$. Such a line is called an ordinary line. Csima and Sawyer [9] improved this result by showing that there are at least $\lceil 6|S| / 13\rceil$ ordinary lines.

We now consider a similar problem on $R \cup B$. A line that passes through at least two red points and no blue points or through at least two blue points and no red points is called a monochromatic line. On the other hand, a line that passes through at least one red point and at least one blue point is called a bichromatic line. A bichromatic ordinary line is a line that passes through exactly one red point and one blue. It is easy to show that there exist configurations $R \cup B$ for which there exists no bichromatic ordinary lines (Figure $14(\mathrm{a})$ ). We beign with a result on monochromatic lines.

Theorem 6.1 (Chakerian [8], [2]). If $R \cup B$ does not lie on a line, then there exists a monochromatic line.

The next theorem deals with the number of bichromatic lines.

Theorem 6.2 (Pach and Pinchasi [35]). Suppose $|R|=|B|=n$. Then there exists more than $n / 2$ bichromatic lines that pass through at most two red points and at most two blue points. Furthermore, the number of bichromatic lines passing through at most six points is at least $m / 10$, where $m$ is the total number of connecting lines.

Conjecture 6.3 (Fukuda [15], [10]). If (i) $R$ and $B$ are separated by a line, and (ii) $|R|$ and $|B|$ differ by at most one, then there exists a bichromatic ordinary line.

Note that the two conditions in the above conjecture are necessary. Namely, Figure 14 (a) and (b) show that condition (i) is necessary, and (c) shows that the condition (ii) is necessary ([35]). Recently, Finschi and Fukuda [13] showed that the above Conjecture 6.3 is true for $|R \cup B| \leq 8$, and found a counter-example to the conjecture, which consists of five red points and four blue points. 


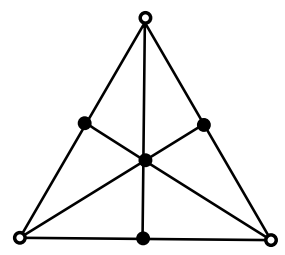

(a)

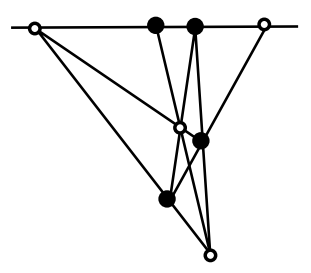

(b)

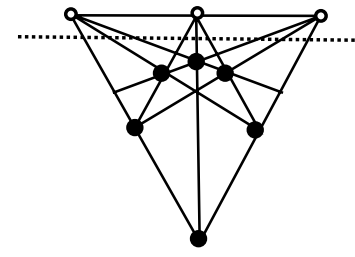

(c)

Figure 14: Configurations having no bichromatic ordinary lines.

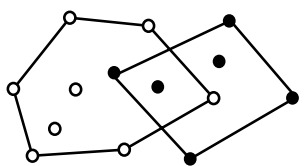

(a)

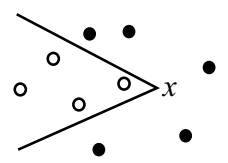

(b)

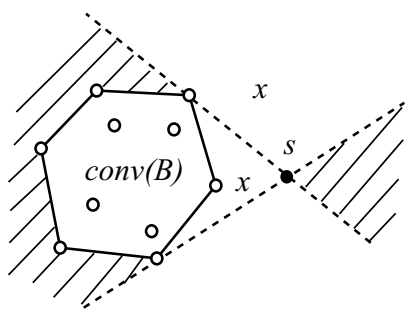

(c)

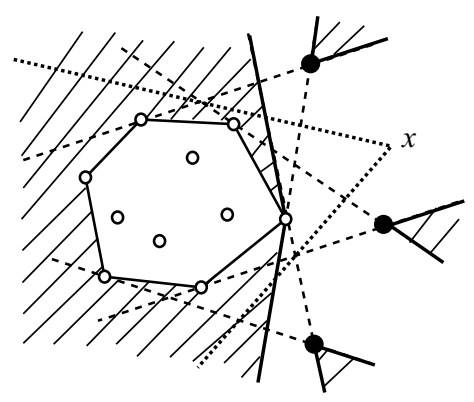

(d)

Figure 15: (A) $R \cup B$ that cannot be separated by a wedge; (b) A point $x$ for which there exists a wedge separating $B$ and $R$; (c) The shaded region Shade $(s)$ with respect $s$; (d) The region of points $x$ for which there exists a wedge separating $B$ and $R$.

We next consider a separation problem. It is obvious that $R$ and $B$ can be separated by a line if and only if $\operatorname{conv}(R) \cap \operatorname{conv}(B)=\emptyset$. It is also easy to see that if $\operatorname{conv}(B)$ contains a red point and if $\operatorname{conv}(R)$ contains a blue point, then $R$ and $B$ cannot be separated by a wedge (Figure 15 (a)). Thus when we consider a wedge-separation, we may assume that $\operatorname{conv}(B)$ contains no red points. Then for a red point $s$, if a point $x$ is not contained in the shaded region in Figure $15(\mathrm{c})$, then there exists a wedge with top $x$ that separates $\operatorname{conv}(B)$ and $s$. By this observation, we can obtain the following theorem.

Theorem 6.4 (Hurtado, Noy, Ramos and Seara [18]). Suppose that conv $(B) \cap R=\emptyset$. For any red point $s \in R$, we denote by Shade(s) the shaded region with respect $s$. Then there exists a wedge with top $x$ that separate $R$ and $B$ if and only if

$$
x \notin \bigcup_{s \in R} \operatorname{Shade}(s) \cup \operatorname{conv}(B)
$$

(Figure $15(d)$ ).

We now turn our attention to a monochromatic partitioning problem. Given a $S=R \cup B$ in general position, let $p(S)$ be the minimum number $k$ of monochromatic subsets $S=X_{1} \cup X_{2} \cup \cdots \cup X_{k}$ such that $\operatorname{conv}\left(X_{i}\right) \cap \operatorname{conv}\left(X_{j}\right)=\emptyset$ for all $i \neq j$. Let

$$
p(n)=\max \{p(S)|| S|=| R \cup B \mid=n\} .
$$

Then $p(n)$ was recently determined as below.

Theorem 6.5 (Dumitrescu and Pach [12]).

$$
p(n)=\left\lceil\frac{n+1}{2}\right\rceil .
$$


The following proposition was posed as a problem in the 27th International Mathematics Olympiad, 1986.

Proposition 6.6. Let $P$ be $n$ points with integer coordinates. Then each point of $P$ can be colored red or blue so that for every row and column, the number of red points on it differs from the number of blue points on it by at most one. This coloring is called a balanced coloring.

This proposition can be proved by using graph theory as follows. Let $X=\left\{x_{1}, \ldots, x_{n}\right\}$ and $Y=$ $\left\{y_{1}, \ldots, y_{m}\right\}$ be the sets of integers such that for every $1 \leq i \leq n$ and $1 \leq j \leq m, P$ contains a point with coordinate $\left(x_{i}, y_{t}\right)$ and a point with coordinate $\left(x_{s}, y_{j}\right)$ for some $y_{t}$ and $x_{s}$. Then we construct the bipartite graph $B=B(X, Y)$ with bipartition $X \cup Y$ in which $x_{i} \in X$ and $y_{j} \in Y$ are joined by an edge if and only the point with coordinate $\left(x_{i}, y_{j}\right)$ is contained in $P$. In particular, the exists a one to one correspondence between the edges of $B$ and the points of $P$. Then by a well-known theorem [32], every edge of $B$ can be colored red and blue so that for every vertex $v$ of $B$, the number of red edges incident with $v$ differs from the number of blue edges incident with $v$ by at most one. This implies the above Proposition 6.6.

Akiyama and Urrutia [3] generalized the above proposition by considering an $m$-coloring, where $m \geq 3$. This generalization also can be obtained by the same arguments as above since a similar result hold for any $m$-edge-coloring of a bipartite graph.

\section{References}

[1] M. Abellanas, J. García, G. Hernández, M. Noy and P. Ramos, Bipartite embeddings of trees in the plane, Discrete Appl. Math. 93 (1999) 141-148.

[2] M. Aigner and G.M. Ziegler, Proofs from the book, 2nd edition, Springer, Berlin, (2001) 63.

[3] J. Akiyama and J. Urrutia, A note on balanced colorings for lattice points, Discrete Math. 83 (1990) $123-126$.

[4] J. Akiyama and J. Urrutia, Simple alternating path problem, Discrete Math. 84 (1990) 101-103.

[5] I. Bárány and J. Matoušek, Simultaneous partitions of measures by $k$-fans, Discrete Comput. Geom. 25 (2001)317-334.

[6] I. Bárány and J. Matoušek, Equipartition of two measures by a 4-fan, preprint.

[7] S. Bespamyatnikh, D. Kirkpatrick and J. Snoeyink, Generalizing ham sandwich cuts to equitable subdivisions, Discrete Comput. Geom. 24 (2000) 605-622.

[8] G.D. Chakerian, Sylvester's problem on collinear points and a relative, Amer. Math. Monthly $\mathbf{7 7}$ (1970) 164-167.

[9] J. Csima and E.T. Sawyer, There exist 6n/13 ordinary points, Discrete Comput. Geom. 9 (1993) $87-202$.

[10] P.F. Da Silva and K. Fukuda, Isolating points by line in the plane, J. Geom. 62 (1998) 48-65.

[11] A. Dumitrescu and R. Kaye, Matching colored points in the plane: Some new results, Comput. Geom. 19 (2001) 69-85.

[12] A. Dumitrescu and J. Pach, Partitioning colored point sets into monochormatic parts, LNCS 2125 (2001), 264-275.

[13] L. Finschi and K. Fukuda, Complete combinatorial generation of small point configurations and hyperplane arrangements, Discrete and Computational Geometry: The Goodman-Pollack Festschrift (in the Series Algorithms and Combinatorics), Springer Verlag, preprint. 
[14] H. de Fraysseix, J. Pach and R. Pollack, How to draw a planar graph on a grid, Combinatorica 10 (1990) 41-51.

[15] K. Fukuda, Question raised at the problem session, in "AMS-IMS-SIAM Joint Summer Research Conference on Discrete and Computational Geometry: Ten Years Later", Mount Holyoke College, South Hadley, Massachusetts, (1996).

[16] T. Gallai, Solution of problem 4065, Amer. Math. Monthly 51 (1944) 179-171.

[17] Handbook of Discrete and Computational Geometry, edited by J. Goodman and J. O'Rourke, CRC Press, (1997) 211

[18] F. Hurtado, M. Noy, P.A. Ramos and C. Seara, Separating objects in the plane by wedges and strips, Discrete Math. 109 (2001) 109-138.

[19] H. Ito, H. Uehara, and M. Yokoyama, 2-dimensional ham-sandwich theorem for partitioning into three convex pieces, Discrete Comput. Geom. LNCS 1763 (2000) 129-157.

[20] Y. Ikeba, M. Perles, A. Tamura and S. Tokunaga, The rooted tree embedding problem into points on the plane, Discrete Comput. Geom. 11 (1994) 51-63.

[21] A. Kaneko and M. Kano, Balanced partitions of two sets of points in the plane, Comput. Geom. 13 (1999) 253-261.

[22] A.Kaneko and M. Kano, Straight-line embeddings of two rooted trees in the plane, Discrete Comput. Geom. 21 (1999) 603-613.

[23] A.Kaneko and M. Kano, Straight line embeddings of rooted star forests in the plane, Discrete Appl. Math. 101 (2000)167-175.

[24] A. Kaneko and M. Kano, Generalized balanced partitions of two sets of points in the plane, Discrete Comput. Geom. LNCS 2098 (2001) 176-186.

[25] A. Kaneko, M. Kano and K. Yoshimoto, Alternating Hamiltonian cycles with minimum number of crossings in the plane, Internat. J. Comput. Geom. Appl., 10 (2000) 73-78.

[26] A. Kaneko and M. Kano, A balanced partition of points in the plane and tree embedding problems, preprint.

[27] A. Kaneko, M. Kano and K. Suzuki Balanced Partitions and Path Covering of Two Sets of Points in the Plane, preprint.

[28] A. Kaneko and M. Kano Certain Balanced Partitions of Two Sets of Points in the Plane, preprint.

[29] A. Kaneko, M. Kano and S. Tokunaga, Straight-line embeddings of three rooted trees in the plane, in Tenth Canadian Conference on Computational Geometry, Extended abstract (1998).

[30] L.C. Larson, Problem-Solving Through Problems, (Springer, New York), (1983) 200-201.

[31] C.-Y. Lo, J. Matouśek, and W. Steiger, Algorithms for ham-sandwich cuts, Discrete Comput. Geom., 11 (1994) 433-452.

[32] L. Lovász, Combinatorial Problems and Exercises, North-Holland, Amsterdam (1979) p.50, Problem 11.

[33] J. Pach, Geometric Graph Theory, Surveys in Combinatorics, 1999, London Math. Soc. Lecture Note Series 267, (1999) 167-200.

[34] J. Pach and P.K. Agarwal, Combinatorial Geometry, Wiley, (1995) 223-242.

[35] J. Pach and R. Pinchasi, Bichromatic lines with few points, J. Combinatorial Theory Ser. A, 90 (2000) 326-335. 
[36] J. Pach and R. Pinchasi, On the number of balanced lines, Discrete Comput. Geom., 25 (2001) $611-628$.

[37] J. Pach and J. Töröcsik, Layout of rooted tree, Planar Graphs (DIMACS Series in Discrete Math. and Theoritical Comput. Sci.) 9 (1993) 131-137.

[38] M. Perles, Open problem proposed at the DIMACS Workshop on Arrangements, Rutgers University, 1990.

[39] T. Sakai, Balanced Convex Partitions of Measures in $R^{2}$, to appear in Graphs and Combinatorics.

[40] J. Sylvester, Mathematical question 11851, Educational Times 59 (1893) 98-99.

[41] S. Tokunaga, On a straight-line embedding problem of graphs, Discrete Math. 150 (1996) 371-378.

[42] S. Tokunaga, Intersection number of two connected geometric graphs Information Proc. Discrete Math. 150 (1996) 371-378.

About Authors

Atsushi Kaneko is at the Department of Computer Science and Communication Engineering, Kogakuin University, Nishi-Shinjuku, Shinjuku-ku, Tokyo 163-8677 Japan; kaneko@ee.kogakuin.ac.jp. M. Kano is at Department of Computer and Information Sciences, Ibaraki University, Hitachi 316-8511 Japan; kano@cis.ibaraki.ac.jp.

\section{Acknowledgments}

Work on this paper has been supported by the Ministry of Education of Japan (the grant-in-aid to Kaneko and Kano). The authors would like to thank a referee for his very careful and helpful suggestions, and the editors for their helpful suggestions and nice contribution. 